\title{
From a discrete to a continuum model of cell dynamics in one dimension
}

\author{
Philip J. Murray, ${ }^{1}$ Carina M. Edwards, ${ }^{2}$ Marcus J. Tindall, ${ }^{3}$ and Philip K. Maini ${ }^{1,4}$ \\ ${ }^{1}$ Centre for Mathematical Biology, Mathematical Institute, 24-29 St. Giles', Oxford OX1 3LB, United Kingdom \\ ${ }^{2}$ Center for Modeling and Simulation in the Biosciences, University of Heidelberg, Im Neuenheimer Feld 267, 69120 Heidelberg, \\ Germany \\ ${ }^{3}$ Institute for Cardiovascular and Metabolic Research and School of Biological Sciences and Department of Mathematics, University of \\ Reading, Whiteknights, Reading, Berkshire RG6 6AJ, United Kingdom \\ ${ }^{4}$ Department of Biochemistry, Oxford Centre for Integrative Systems Biology, South Parks Road, Oxford OX1 3QU, United Kingdom
}

(Received 25 March 2009; published 23 September 2009)

\begin{abstract}
Multiscale modeling is emerging as one of the key challenges in mathematical biology. However, the recent rapid increase in the number of modeling methodologies being used to describe cell populations has raised a number of interesting questions. For example, at the cellular scale, how can the appropriate discrete cell-level model be identified in a given context? Additionally, how can the many phenomenological assumptions used in the derivation of models at the continuum scale be related to individual cell behavior? In order to begin to address such questions, we consider a discrete one-dimensional cell-based model in which cells are assumed to interact via linear springs. From the discrete equations of motion, the continuous Rouse [P. E. Rouse, J. Chem. Phys. 21, 1272 (1953)] model is obtained. This formalism readily allows the definition of a cell number density for which a nonlinear "fast" diffusion equation is derived. Excellent agreement is demonstrated between the continuum and discrete models. Subsequently, via the incorporation of cell division, we demonstrate that the derived nonlinear diffusion model is robust to the inclusion of more realistic biological detail. In the limit of stiff springs, where cells can be considered to be incompressible, we show that cell velocity can be directly related to cell production. This assumption is frequently made in the literature but our derivation places limits on its validity. Finally, the model is compared with a model of a similar form recently derived for a different discrete cell-based model and it is shown how the different diffusion coefficients can be understood in terms of the underlying assumptions about cell behavior in the respective discrete models.
\end{abstract}

DOI: 10.1103/PhysRevE.80.031912

PACS number(s): 87.17.Aa, 87.17.Ee, 87.17.Rt

\section{INTRODUCTION}

The mathematical modeling of cell populations can be, broadly speaking, split into two categories: continuum and discrete models. Discrete models treat cells as individual entities and hence provide a natural framework within which to make use of an increasing amount of experimental data available at the cellular and subcellular scales. There are now many different types of discrete cell-level models used to describe cell populations, e.g., cellular automata [1-3], cellular Potts models [4,5], cell-vertex [6] and off-lattice cellbased models [7-10].

Often, however, observed behavior occurs at the continuum rather than cellular scale. Therefore, at the scale of observation it is often satisfactory to treat a cell population as a continuum-an average over individual cells. This is one of the reasons why there is a strong historical usage of continuum models that describe cell populations [11-16].

Continuum and discrete models address the model building problem from different perspectives and depending on the question being asked, one or the other (or both) may be the appropriate model framework to choose for a particular problem. The two modeling paradigms have complimentary strengths and weaknesses: continuum models are suitable for describing large populations of cells, while discrete models are often computationally intractable in this regime; discrete models can be related to subcellular mechanisms and data, while continuum models are often derived making assumptions that are difficult to directly relate to individual cell behavior; and continuum model solutions can be expressed in terms of model parameters, while discrete models typically require many computationally intensive simulations to develop insight into system-level behavior. However, by deriving continuum models directly from their discrete counterparts, many of the above-mentioned problems of using discrete models can be overcome.

Discrete, off-lattice, cell-based models have been used to model epithelial cell motion in intestinal crypts (e.g., $[9,17])$ and tumor monolayer and spheroid growth (e.g., $[7,10,18,19])$. Perhaps the simplest assumption that captures the fundamental properties of cell elasticity and adhesion is that a pair of neighboring cells is connected via a linear spring (e.g., $[9,17]$ ), hence cells seek to maintain a fixed distance (the resting spring length) between one another. In this paper we consider the case of a one-dimensional (1D) off-lattice cell-based model in which cells interact via linear springs and show that in the continuum limit, and after making a suitable coordinate transformation, cell number density, $q(r, \tau)$, evolves according to

$$
\frac{\partial q}{\partial \tau}=\frac{\partial}{\partial r}\left(\frac{k}{\eta q^{2}} \frac{\partial q}{\partial r}\right)
$$

where $k$ is the spring constant, $\eta$ is the cell viscosity, $\tau$ is time, and $r$ is the spatial coordinate. We define the nonlinear diffusion coefficient $D(q)=k / \eta q^{2}$.

The nonlinear diffusion coefficient corresponds to a case of "fast" diffusion and has been considered previously as a 
model for macroscopic diffusion [20,21]. However, the derivation in this paper explicitly relates the phenomenon of macroscopic diffusion to parameters and processes at the microscale. The form of our derived diffusion coefficient is in contrast with mean-field derived models where a constant diffusion coefficient is typically related to stochastic features of the cell dynamics. For example, Newman [22] has shown that an advection-diffusion equation for cell density can be derived from a subcellular element model using mean-field approximations. The diffusion coefficient scales with the noise strength in the discrete dynamics while the advection term is dependent on an intercellular potential.

The nonlinear diffusion coefficient derived in this paper bears significant resemblance to that obtained by Lushnikov et al. [23], who coarse grained an on-lattice cellular Potts model in order to derive a partial differential equation (PDE) for cell number density. Their work follows from an initial study by Turner et al. [24] who, considering a population of noninteracting cells, derived a macroscopic diffusion coefficient in terms of microscale parameters. The differing forms for the diffusion coefficients allow one to differentiate between the underlying cell-based models and to determine which cell-based models are most suited to a particular modeling problem.

The layout of the rest of this paper is as follows: in Sec. II we present details of the one-dimensional cell-based model and derive a linear diffusion equation in the continuum limit. In Sec. III we consider a coordinate transformation and subsequently derive the nonlinear diffusion equation presented in Eq. (1). In Sec. IV we compare simulations of the cellbased model with numerical solutions of the nonlinear diffusion equation. In Sec. V we consider applications of the derived nonlinear diffusion model; in Sec. V A we introduce the process of cell division and show excellent agreement between continuum and discrete models. In Sec. V B we demonstrate how using the adiabatic approximation the nonlinear diffusion model yields an approximation commonly made in the modeling of cell populations. In Sec. V C we analyze the derived diffusion coefficient and compare results with those from Alber and co-workers [5,23]. In Sec. VI we summarize the main results in this paper and discuss future directions.

\section{MICROSCOPIC CELL MOTION}

Newton's second law of motion can be defined for the $i$ th cell in a population to be

$$
m_{i} \frac{d^{2} \mathbf{r}_{i}}{d t^{2}}=\sum_{j \neq i} \mathbf{F}_{i j}^{i n t}+\mathbf{F}_{i}^{V i s c},
$$

where $m_{i}$ is the mass of the $i$ th cell, $\mathbf{r}_{i}$ is its spatial position, $\mathbf{F}_{i j}^{i n t}$ is the interaction force between a pair of neighboring cells, $\mathbf{F}_{i}^{V i s c}$ is the viscous force acting on the $i$ th cell, and the sum is taken over neighboring cells. Note that in this paper we consider unit length to be a cell diameter $\left[O\left(10^{-5}\right) \mathrm{m}\right]$ while time is measured in hours.

A number of assumptions can be made that simplify Eq. (2). First, we consider a one-dimensional chain with $N$ cells,

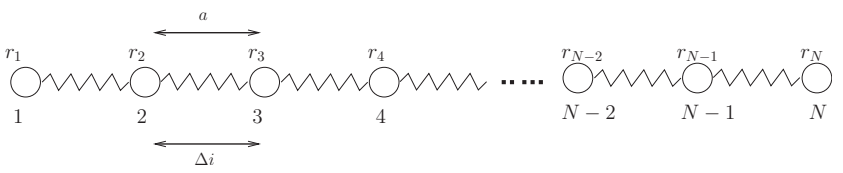

FIG. 1. A schematic of a one-dimensional cell-based model. A one-dimensional chain of cells with cell positions, $r_{i}(t)$, and cell labeling indices, $i$, is depicted. In this schematic, the springs are in equilibrium hence the distance between cells is the resting spring length $a$. Note that cell labeling indices increase with distance from $r_{1}(t)$. Here $\Delta i$ denotes the distance between cells along the indexing coordinate.

as illustrated in Fig. 1, where it is assumed that the first cell is fixed $\left(r_{1}=0\right)$ while the $N$ th cell is attached only to the $(N-1)$ st cell. Each interior cell in the one-dimensional chain has two neighbors. Second, we model the interaction force as being linear, hence using Hooke's law (as in Meineke et al. [9]), the force, $\mathbf{F}_{i j}^{i n t}$, exerted on the $i$ th cell by the $j$ th cell is

$$
\mathbf{F}_{i j}^{i n t}=-k\left(\left|\mathbf{r}_{i}-\mathbf{r}_{j}\right|-a\right) \frac{\mathbf{r}_{i}-\mathbf{r}_{j}}{\left|\mathbf{r}_{i}-\mathbf{r}_{j}\right|} .
$$

Third, the viscous force, which is generated by a combination of cell-cell, cell-medium and cell-matrix interactions, is modeled by assuming that the drag on the $i$ th cell is independent of the springs and is proportional to its velocity $[9,25]$, with the constant of proportionality (the cell viscosity coefficient) given by $\eta$. Fourth, as cells typically move in relatively dissipative environments, motion can be approximated as being overdamped $[9,10,18]$, hence $m_{i} d^{2} \mathbf{r}_{i} / d t^{2} \sim 0$. Therefore, Eq. (2) can be approximated as being a first-order equation of the form

$$
\frac{d r_{i}(t)}{d t}=\alpha\left[r_{i-1}(t)-2 r_{i}(t)+r_{i+1}(t)\right], \quad i=2, \ldots, N-1,
$$

where $\alpha=\frac{k}{\eta}$. $\alpha$ will take different values in different tissues and in Sec. VI we describe how it could be measured experimentally. We note that in the case of epithelial cells in an intestinal crypt, it has been estimated, based on analysis of numerical simulations, by Meineke et al. [9] to be $36 \mathrm{hr}^{-1}$. The equation of motion for the $N$ th cell is

$$
\frac{d r_{N}(t)}{d t}=\alpha\left[r_{N-1}(t)-r_{N}(t)+a\right],
$$

and the first cell $(i=1)$ is pinned at the origin, hence

$$
r_{1}(t)=0 \text {. }
$$

Equation (4) is of a similar form to a standard model for polymer chain dynamics developed by Rouse [26]. For long chains with large $N$, the continuum approximation of this model is

$$
\frac{\partial r}{\partial t}=\alpha \frac{\partial^{2} r}{\partial i^{2}}, \quad i \in[1, N],
$$

see, for example, [27,28], where $r$ is now a continuous function of $i$ such that 


$$
r(i, t)=r_{i}(t)
$$

This approach can be justified by noting that the right-hand side of Eq. (4) looks like the discretization of the second derivative of $r$ with respect to $i$,

$$
\alpha \frac{\left[r_{i-\Delta i}(t)-2 r_{i}(t)+r_{i+\Delta i}(t)\right]}{\Delta i^{2}},
$$

in the special case where $\Delta i=1$. The $1 / \Delta i^{2}$ term in the denominator arises as the drag coefficient scales linearly with $\Delta i$, the number of springs being averaged over, while the effective spring constant scales with $\frac{1}{\Delta i}$ so that $\alpha$ scales like $1 / \Delta i^{2}$. Similarly, the resting spring length $a$ scales with $\Delta i$.

The boundary condition at $i=1$ is simply

$$
r(1)=0,
$$

and by introducing an image cell at position $i=N+1$ such that $r(N+1)=r(N)+a$, the motion of the $N$ th cell is captured. In the continuum limit this leads to the boundary condition

$$
\left.\frac{\partial r}{\partial i}\right|_{i=N}=a \text {. }
$$

Together with the initial condition

$$
r(i, 0)=r_{0}(i)
$$

and boundary conditions (10) and (11), the solution of the linear Eq. (7), found using separation of variables, is in excellent agreement with simulation results from the discrete cell-based model (results not presented).

\section{MACROSCOPIC MOTION}

In order that Eq. (4) can be interpreted as a diffusion equation, we require that the number of cells is large enough to define a number density, $q(r, t)$, of cells per unit length, so that the variable $i$ may be interpreted as

$$
i(r, t)=\int_{0}^{r} q\left(r^{\prime}, t\right) d r^{\prime} .
$$

In a coordinate system where the length scale of the problem is the resting spring length, we therefore expect $q(r, t)$ to be an $O(1)$ quantity for biologically relevant problems. Equation (7) can be reformulated such that cell number density is the dependent variable by making a coordinate transformation from the old independent variables $i$ and $t$ to the new independent variables $r$ (the dependent variable in the old coordinate system) and $\tau$ (time). The Jacobian of the coordinate transformation is

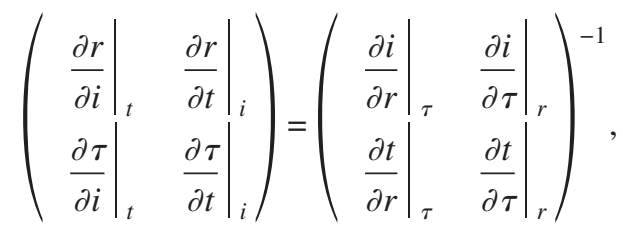

and upon letting $t=\tau$ we can read off the relationships

$$
\left.\frac{\partial r}{\partial i}\right|_{t}=\frac{1}{\left.\frac{\partial i}{\partial r}\right|_{\tau}}
$$

and

$$
\left.\frac{\partial r}{\partial t}\right|_{i}=-\left.\frac{\left.\frac{\partial i}{\partial \tau}\right|_{r}}{\frac{\partial i}{\partial r}}\right|_{\tau}
$$

Upon rearrangement, Eq. (7) can be written as

$$
\frac{\partial r}{\partial t}=\alpha \frac{\partial}{\partial i}\left(\frac{\partial r}{\partial i}\right)
$$

which becomes

$$
-\frac{1}{\frac{\partial i}{\partial r}} \frac{\partial i}{\partial \tau}=\alpha \frac{1}{\frac{\partial i}{\partial r}} \frac{\partial}{\partial r}\left(\frac{1}{\frac{\partial i}{\partial r}}\right)
$$

Differentiating through on the right-hand side using the chain rule and cancelling terms yields

$$
\frac{\partial i}{\partial \tau}=\alpha\left(\frac{\partial i}{\partial r}\right)^{-2} \frac{\partial}{\partial r}\left(\frac{\partial i}{\partial r}\right) .
$$

We now differentiate both sides of Eq. (19) with respect to $r$ and, swapping the order of the derivatives on the left-hand side, obtain

$$
\frac{\partial}{\partial \tau}\left(\frac{\partial i}{\partial r}\right)=\alpha \frac{\partial}{\partial r}\left[\left(\frac{\partial i}{\partial r}\right)^{-2} \frac{\partial}{\partial r}\left(\frac{\partial i}{\partial r}\right)\right] .
$$

Finally, noting that differentiation of Eq. (13) with respect to $r$ yields

$$
q(r, \tau)=\frac{\partial i(r, \tau)}{\partial r},
$$

Eq. (20) can be rewritten as

$$
\frac{\partial q}{\partial \tau}=\frac{\partial}{\partial r}\left(\frac{\alpha}{q^{2}} \frac{\partial q}{\partial r}\right) .
$$

Defining $D(q)=\frac{\alpha}{q^{2}}$, the evolution equation for $q(r, \tau)$ is

$$
\frac{\partial q}{\partial \tau}=\frac{\partial}{\partial r}\left[D(q) \frac{\partial q}{\partial r}\right]
$$

which is of the form presented in Eq. (1).

Boundary condition (10) can be reformulated by introducing an image cell at $i=0$ such that $r(0)=-r(2)$. Upon treating the cell at $i=1$ as an interior cell, the condition on the image cell ensures that the cell at $i=1$ remains stationary as the net force on it is zero (its neighbors are equidistant from it). This condition implies that $r$ is a linearly increasing function of $i$ at $i=1$ and therefore that

$$
\left.\frac{\partial^{2} r}{\partial i^{2}}\right|_{i=1}=0
$$

Upon substitution for $\frac{\partial r}{\partial i}$ and $\frac{\partial}{\partial i}$ we obtain 


$$
\left.\frac{\partial q}{\partial r}\right|_{r=0}=0,
$$

which is simply a no-flux condition, as expected, for the cell number density at the origin. In the old coordinate system both the boundaries $i=1$ and $i=N$ were fixed as $N$, the total cell number, was constant. However, in the new coordinate system, the spatial position of the $N$ th cell varies in time, hence we have a moving boundary problem. Invoking conservation of cell number, it is straightforward to show that the moving boundary, $S(\tau)$, must move with the local cell velocity

$$
\frac{d S}{d \tau}=-\left.\frac{D(q)}{q} \frac{\partial q}{\partial r}\right|_{r=S(\tau)} .
$$

Using Eq. (15) to substitute for $\frac{\partial r}{\partial i}$ in Eq. (11) gives

$$
q[S(\tau), \tau]=\frac{1}{a},
$$

which can be understood as a cell at the boundary, $S(\tau)$, experiencing a force only from cells in the interior, i.e., for $r>S(\tau)$, the cell number density is effectively the equilibrium cell number density. The initial cell number density is defined to be

$$
q_{0}(r)=q(r, 0),
$$

which for the purposes of comparing continuum and discrete models will be determined by the initial conditions in a given simulation. Equations (23)-(28) constitute a system of closed equations which we solve numerically after defining the effective cell velocity field

$$
v(r, \tau)=-\frac{D(q)}{q} \frac{\partial q}{\partial r},
$$

which after differentiation with respect to $r$ yields

$$
\frac{\partial v}{\partial r}=-\frac{D(q)}{q}\left[\frac{\partial^{2} q}{\partial r^{2}}-\frac{3}{q}\left(\frac{\partial q}{\partial r}\right)^{2}\right] .
$$

Given the effective velocity field $v(r, \tau)$, Eq. (23) can be rewritten using the material derivative, $\frac{D q}{D \tau}$, such that

$$
\frac{D q}{D \tau}=\frac{\partial q}{\partial \tau}+v \frac{\partial q}{\partial r}=-q \frac{\partial v}{\partial r} .
$$

However, Eq. (31) describes how the solution $q(r, \tau)$ changes at a point $r$ which moves with a velocity

$$
\frac{d r}{d \tau}=v(r, \tau),
$$

hence the evolution of the moving boundary, $S(\tau)$, defined by Eq. (26), is captured by Eq. (32). Numerical solutions of Eqs. (29)-(32) were computed using the method of lines with a central difference stencil used to approximate the spatial derivative in Eq. (30). The resulting set of nonlinear ordinary differential equations was solved using the MATLAB solver "ODE 45 " on a regularly discretized initial domain $\left[0, S_{0}\right]$ with $N_{\text {Nod }}$ nodes.
We note that coordinate transformations are typically made in order to simplify the mathematical description of a problem rather than to make it more complex. However, in this section we have made a coordinate transformation that maps a linear diffusion equation defined on a fixed domain onto a nonlinear diffusion equation defined on a varying domain. This step can be justified by noting that in the new coordinate system, the cell-based model is described in terms of a cell number density, which is standard in the cell population modeling literature (e.g., [15]).

\section{COMPARISON OF CELL-BASED AND CONTINUUM MODELS}

In order to compare numerical solutions of the onedimensional continuum and cell-based models, we now consider an illustrative example in which the initial cell density distribution is Gaussian and given by

$$
q_{0}(r)=\frac{n_{0}}{\sqrt{2 \pi} \sigma} e^{-(r-\mu)^{2} / 2 \sigma^{2}}, \quad 0 \leq r \leq 300,
$$

where $\sigma=100, \mu=160.5$, and the initial number of cells, $n_{0}$, was 700. In Fig. 2 cell number density (left column) and velocity (right column) are plotted against radial coordinate at a sequence of times. The bars depict data from a simulation of the cell-based model while the solid lines denote numerical solutions of Eqs. (23)-(28). The peak in the initial distribution at $r=\mu=160.5$ induces an initial negative velocity for $r<\mu$ and a positive velocity for $r>\mu$. In the region $r<\mu$, the zero flux boundary condition at $r=0$ results in the cell number density initially increasing, while for $r>\mu$, cells extend outward and the boundary condition at $r=S(\tau)$ is satisfied. Note that $v(r, \tau)=0$ corresponds to $\frac{\partial q}{\partial r}=0$. The system eventually equilibrates such that $q(r, \tau)=\frac{1}{a}$ for all $r<S(\tau)$ (results not shown). In Fig. 3 a plot of $S(\tau)$ against time verifies that the one-dimensional continuum model (solid line) accurately describes the relaxation toward equilibrium of the moving boundary. The total cell number, $n(\tau)$, is constant in time (results not shown).

In the following section we consider applications of the derived continuum model. In Sec. V A we will consider a simple model for the process of cell division in the discrete model and demonstrate that the nonlinear diffusion coefficient together with an appropriately chosen source term can adequately describe the resulting cell population dynamics; in Sec. V B examine the limit of stiff springs and demonstrate that a phenomenological assumption made in many continuum models is retrieved; and in Sec. V C contrast the diffusion coefficient arising from the cell-based model with that obtained by Alber and co-workers [5,23], who considered a cellular Potts model.

\section{APPLICATIONS}

\section{A. Proliferating cell population}

A valid question to ask is whether the nonlinear diffusion model is robust to the inclusion of more realistic biological effects such as cell proliferation. We now begin to address 

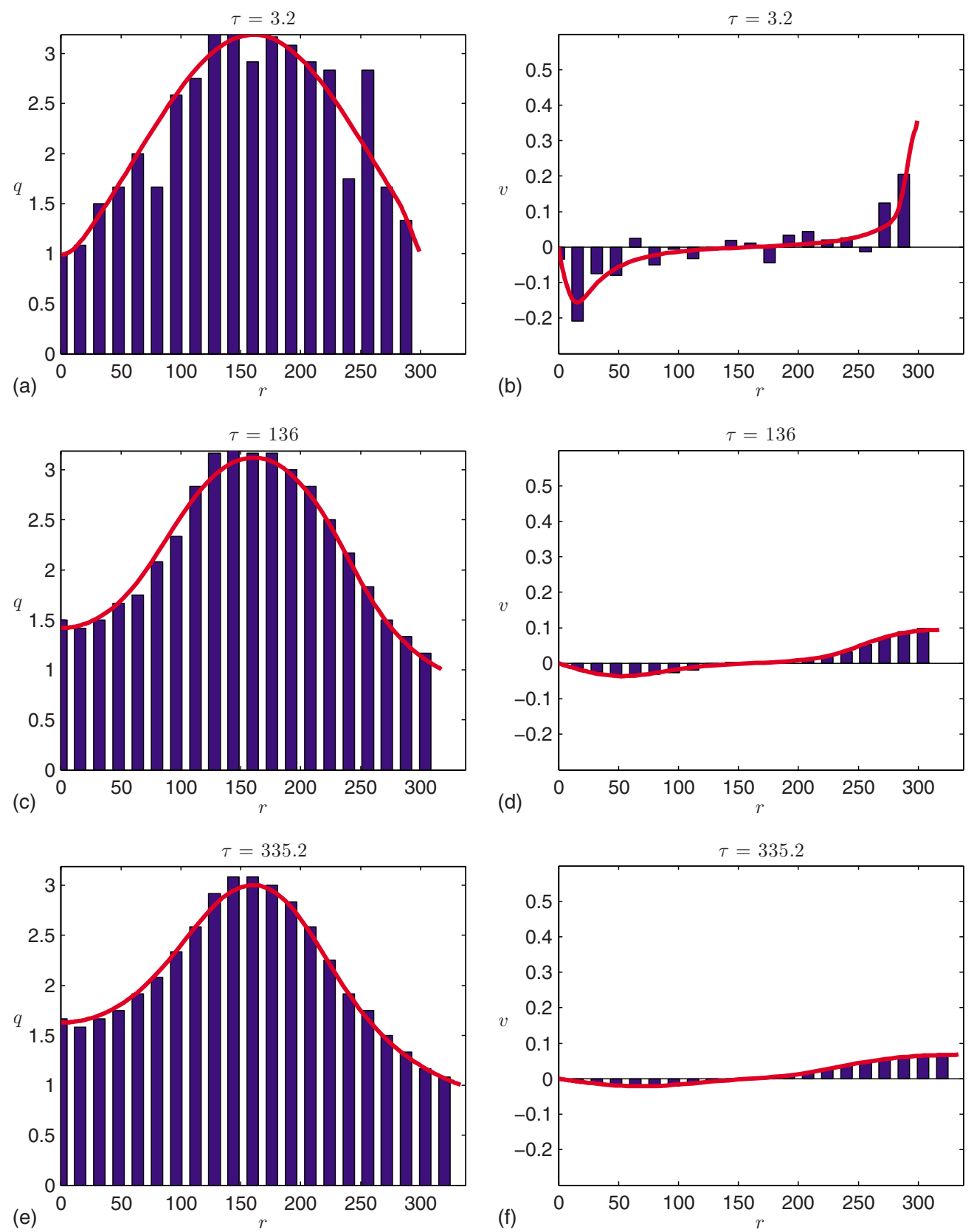

FIG. 2. (Color online) Continuum (solid lines) and one-dimensional cell-based models (bars) are compared as an initially Gaussian distributed cell population relaxes to equilibrium. Cell number density, $q(r, \tau)$, (left column) and velocity, $v(r, \tau)$, (right column) are plotted against $r$ at a sequence of times (hour). Numerical solutions of Eqs. (23)-(28) (lines) were computed with $N_{\text {Nod }}=100, \alpha=15 \mathrm{hr}^{-1}, S_{0}$ $=300$, and $n_{0}=700$.

this question by considering cell-based model simulations in which cells undergo cell division and compare simulation results with the nonlinear diffusion model in which a source term has been included.

We consider a simple model for cell division in which each cell in the simulation divides when its age reaches $T_{C}$. We initially assign the age of the $i$ th cell by randomly drawing from the range $\left[a_{1}, T_{C}\right]$. By choosing $a_{1}=0$, there is no initial age heterogeneity within the cell population. Alternatively, by choosing $a_{1}=T_{C}-1$ (say), all the cells in the cellbased model will divide within the first hour of the simulation and then there will be no further division for $T_{C}-1$ hours. We consider this latter case to be the case where cells divide synchronously. Upon cell division in the 1D simulations, the mother and daughter cells are placed a distance of 0.1 cell diameters on either side of the dividing mother cell. We note that the process of cell growth is not captured explicitly in the discrete simulations but rather through the relaxation from post-cell birth compression.

One of the advantages of developing the model with cell number density as the dependent variable is that it is relatively easy to introduce a cell production term into the governing equation. Defining $f(q, \tau)$ to be a generic source term, Eq. (23) takes the form 


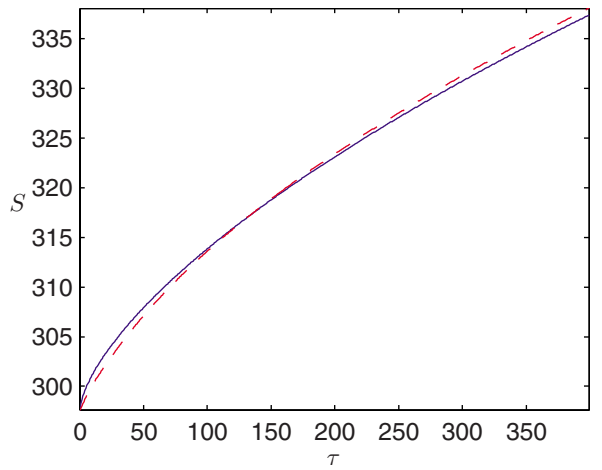

FIG. 3. (Color online) A plot of $S(\tau)$ against time (hour) for the simulation shown in Fig. 2. The solution from the one-dimensional continuum model (solid line) is compared with that from the onedimensional cell-based model (dashed line).

$$
\frac{\partial q}{\partial \tau}=\frac{\partial}{\partial r}\left[D(q) \frac{\partial q}{\partial r}\right]+f(q, \tau) .
$$

For the simple rules of cell division in the discrete model we let

$$
f(q, \tau)=H\left(\tau \bmod T_{C}-a_{1}\right) \frac{\ln 2}{T_{C}-a_{1}} q
$$

where $H(\cdot)$ is a Heaviside function. Note that cell proliferation occurs over a time period $T_{C}-a_{1}$ and that the strength of the source is chosen such that total cell number has doubled every $T_{C}$ hours, i.e., every cell (with cell cycle period $T_{C}$ ) has divided once. The function $f(q, \tau)$ therefore captures the process of tissue growth via an increase in cell number density resulting in compressed springs, which upon relaxation mimics tissue growth.

A comparison of results from continuum and discrete models when $a_{1}=0$ is presented in Fig. 4 . In the left and right columns, cell number density and velocity, respectively, are plotted against $r$. The time scale over which diffusion acts is much greater than that over which division occurs, hence within the population interior, the cell number density increases steadily as time evolves.

However, the boundary condition at $r=S(\tau)$ defines $q[S(\tau), \tau]=\frac{1}{a}$ hence $\frac{\partial q}{\partial r}$ is large near $r=S(\tau)$. As the velocity (right column) is a function of $\frac{\partial q}{\partial r}$, a large velocity is observed close to the boundary $r=S(\tau)$. Note that if the spring constant, $k$, was chosen to be large enough, instead of cell division causing an increase in cell number density in the interior, the moving boundary $S(\tau)$ would extend at a fast enough rate to maintain $q(r, \tau) \sim \frac{1}{a}$. We elaborate further on this point in Sec. V B.

In Fig. 5(a), the moving boundary calculated using the numerical solution of Eq. (26) (solid line) is compared with the position of the outermost cell from the one-dimensional cell-based simulation (dashed line). Clearly the continuum model is capturing gross behavior in the cell-based model. In Fig. 5(b), total cell number is compared with data from the simulation of a one-dimensional cell-based model.
We again observe excellent agreement between continuum and discrete models. Similar agreement to that presented in Fig. 4 is observed when $a_{1} \neq 0$. In Figs. 6(a) and 6(b), $S(\tau)$ and $n(\tau)$, respectively, are plotted against time for the case of synchronized division occurring over a period of $1 \mathrm{~h}\left(a_{1}=11\right.$ and $\left.T_{C}=12\right)$. We note that when cell production is periodic, the cell number increases in a stepwise manner but that as a result of the compressibility of the cells, the moving boundary extends over a longer time scale.

To summarize, we have considered a toy model of cell division in order to demonstrate that the nonlinear diffusion model is a robust description of cell motion when the process of cell proliferation is included. This result suggests that the nonlinear diffusion model provides a framework in which to construct more realistic models of cell proliferation (e.g., models in which cell proliferation depends on extracellular factors such as nutrient concentration or cellular compression).

\section{B. Limit of incompressible cells}

A common assumption made in the tumor modeling literature (e.g., $[14,16])$ is that the density of cellular material is constant (i.e., $q \equiv q_{0}$ ) and hence that

$$
q_{0} \nabla \cdot v=f,
$$

where $f$ is the net cell production rate. This equation can be written in integral form by taking a volume integral of Eq. (36) yielding

$$
q_{0} \frac{d S}{d \tau}=\int_{\Omega} f d V
$$

where it has been assumed that a no-flux condition holds at the origin. We will now show how Eq. (37) can be retrieved from the nonlinear diffusion model.

We nondimensionalize Eq. (34) as follows:

$$
\hat{r}=\frac{r}{L}, \quad \hat{\tau}=\frac{\tau}{T}, \quad \hat{f}=\frac{f T_{C}}{Q}, \quad \hat{q}=\frac{q}{Q},
$$

where $T, L$, and $Q$ are undetermined length, time, and cell number density scales, respectively, and $T_{C}$ is the time scale over which cell division occurs. Upon substitution in Eq. (34) and dropping the hatted notation,

$$
\frac{\partial q}{\partial \tau}=\frac{\alpha T}{L^{2} Q^{2}} \frac{\partial}{\partial r}\left(\frac{1}{q^{2}} \frac{\partial q}{\partial r}\right)+f \frac{T}{T_{C}} .
$$

Choosing the time scale to be the cell production time scale, $T=T_{C}$, and the cell number density scale to be the equilibrium value, $Q=\frac{1}{a}$,

$$
\frac{\partial q}{\partial \tau}=\frac{\alpha T_{C} a^{2}}{L^{2}} \frac{\partial}{\partial r}\left(\frac{1}{q^{2}} \frac{\partial q}{\partial r}\right)+f(q, \tau) .
$$

The diffusive length scale, $L_{D}$, is defined to be

$$
L_{D}=\sqrt{\alpha T_{C} a^{2}},
$$

hence Eq. (40) can be written as 

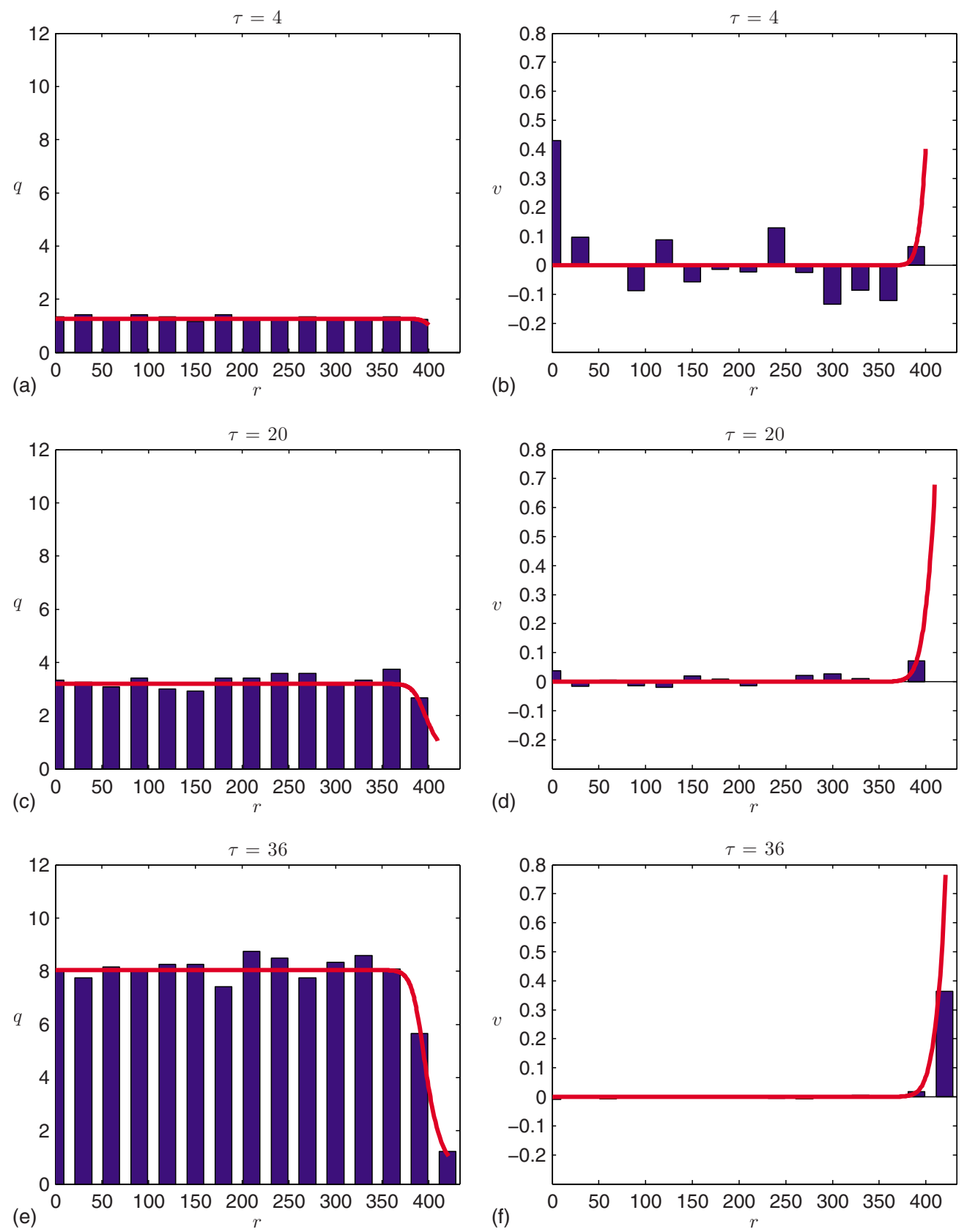

FIG. 4. (Color online) Continuum (solid lines) and discrete (bars) models are compared in the case of a continuously growing cell population $\left(a_{1}=0\right)$. Cell number density, $q(r, \tau)$, (left column) and velocity, $v(r, \tau)$, (right column) are plotted against $r$ at a sequence of times (hour). Initial conditions are $q_{0}(r)=1$ and $S_{0}=399 . T_{C}=12, N_{N o d}=500, \alpha=15 \mathrm{hr}^{-1}$, and $n_{0}=400$.

$$
\frac{\partial q}{\partial \tau}=\frac{L_{D}^{2}}{L^{2}} \frac{\partial}{\partial r}\left(\frac{1}{q^{2}} \frac{\partial q}{\partial r}\right)+f(q, \tau) .
$$

In the limit where $L_{D} \gg L$, corresponding to very stiff springs, the solution to leading order is given by

$$
q(r, \tau) \sim \frac{1}{a}
$$

which corresponds to all springs having resting spring length. An ordinary differential equation for the evolution of total cell number, $n(\tau)$, can be found upon integration of Eq. (42) over the spatial domain $[0, S(\tau)]$ to be

$$
\frac{d n}{d \tau}=\frac{d}{d \tau} \int_{0}^{S(\tau)} q d V=\int_{0}^{S(\tau)} f d V
$$

Using the leading order approximation determined in Eq. (43) we therefore obtain

$$
\frac{1}{a} \frac{d S}{d \tau}=\int_{0}^{S(\tau)} f d V,
$$

as required.

The key point to note from this analysis is that we had to assume that $L_{D} \gg L$. However, if it is assumed that the source 

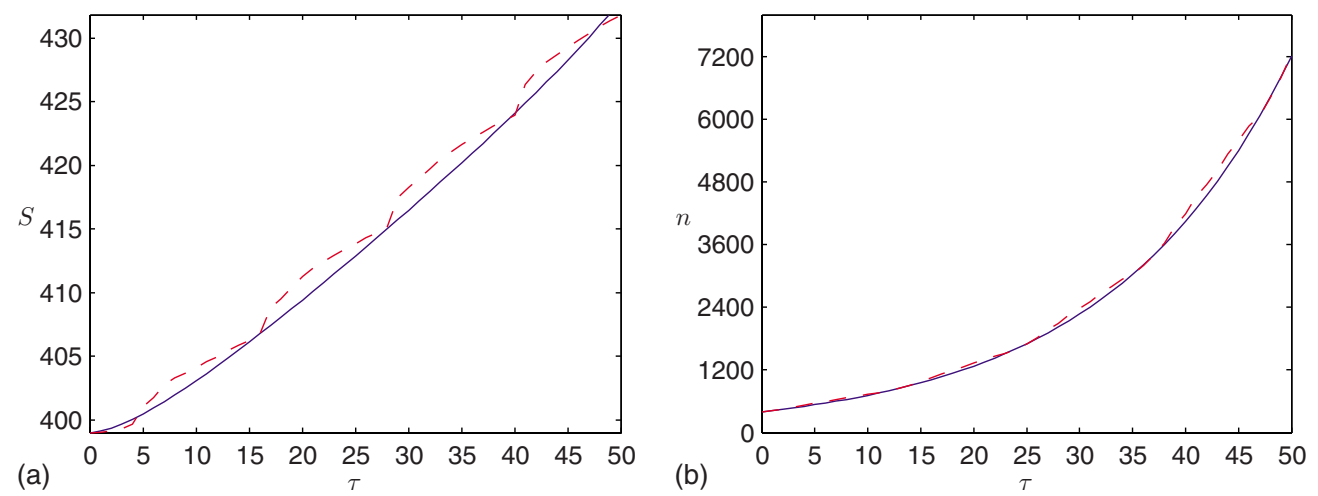

FIG. 5. (Color online) Continuum and discrete models are compared in the case of a continuously growing cell population $\left(a_{1}=0\right)$. The position of the moving boundary, $S(\tau)$, and cell number, $n(\tau)$, are plotted against time (hour).

term, $f(q, \tau)$, is independent of time, as $q$ is constant to lowest order, Eq. (45) states that the moving boundary, $S(\tau)$, evolves according to

$$
\frac{d S(\tau)}{d \tau} \propto S(\tau),
$$

hence the length scale of the problem is an exponentially increasing function of time. Therefore for any finite value of $\alpha$, the assumption that $L_{D} \gg L$ will eventually break down; diffusion will not be able to homogenize the spatial distribution of cells over a time scale $T_{C}$ and the cell number density will exhibit a spatially varying profile.

In this section an assumption made in many continuum models of tumor growth has been retrieved from the nonlinear diffusion model. The key point to note is that limits of validity have been placed on this assumption which are directly related to behavior at the cellular scale.

\section{Model classification}

Our understanding of the nonlinear diffusion coefficient, $D(q)$, can be enhanced by considering the limits of large and small $q$. As $q \rightarrow 0, D(q) \rightarrow \infty$. In the cell-based model this corresponds to the springs connecting cells being extended, a large restoring force drawing cells together and therefore a large diffusion coefficient. Conversely, as $q \rightarrow \infty, D \rightarrow 0$. In the cell-based model, the internal springs are densely packed and far from equilibrium. As there is no space for them to equilibrate, they remain compressed until cells at the boundary have extended outward, freeing up space for the internal cells to equilibrate. From this simple analysis it is clear that the behavior of the discrete model can be adequately described by consideration of the corresponding nonlinear diffusion coefficient.

Lushnikov et al. [23] have considered a cellular Potts model in which cell volume is constrained and cells interact via a hard-core potential. The discrete model is solved using a classical Monte Carlo algorithm. They derive the following nonlinear diffusion coefficient in the macroscopic limit:

$$
D_{A}(q)=C \frac{1+\left(\frac{q}{q_{0}}\right)^{2}}{\left(1-\frac{q}{q_{0}}\right)^{2}},
$$

where $C$ is a constant and $q_{0}$ is a limiting cell number density ( $q_{0}=1 / L_{0}$ in [23] where $L_{0}$ is the average cell length).

As $q \rightarrow 0, D_{A} \rightarrow C$. In this limit cell densities are low and cells behave like random walkers in the cellular Potts model, hence the constant diffusion coefficient. This behavior is in contrast to the low density behavior derived in the springbased model.
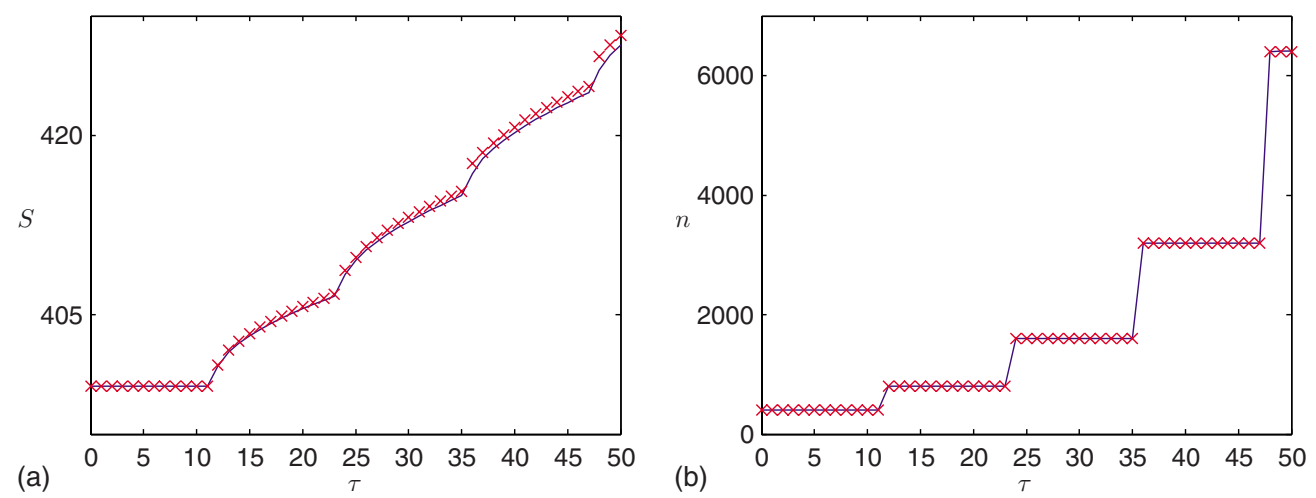

FIG. 6. (Color online) Continuum (solid lines) and one-dimensional cell-based model (crosses) results are compared for the case of a periodically growing cell population. Moving boundary, $S(\tau)$, and total cell number, $n(\tau)$, are plotted against time (hour). Parameters as in Fig. 4. 
In the model of Lushnikov et al. [23] the cell number density $q=q_{0}$ represents the limiting packing density which occurs as a result of the hard-core nature of the cell's interaction potential. As $q \rightarrow q_{0}, D_{A} \rightarrow \infty$. Again, this behavior is in contrast to the diffusion coefficient derived in this paper where $D \rightarrow 0$ as $q$ becomes large.

An interesting point to note when contrasting the different diffusion models is that Lushnikov and co-workers have considered the stochastic cellular Potts model and made scaling and other assumptions in order to finally obtain a nonlinear diffusion coefficient. In contrast, we have considered a deterministic set of equations of motion and used cell indices to define a cell number density and its spatiotemporal evolution. The disparity between diffusion coefficients is merely a reflection of the underlying behavior of the discrete models. In particular, the model derived in this paper assumes that cells are always in contact with each other and hence could be used to describe cell populations in epithelial sheets while the Lushnikov et al. [23] model is perhaps more appropriate for mesenchymal movement.

The process of deriving macroscopic diffusion coefficients permits the classification of cell behavior. By considering the diffusion coefficients in appropriate limits, the differences between cell-level models can be clearly understood. This clarification could allow one to determine which cell-level model may be the appropriate model for a given problem.

\section{SUMMARY AND DISCUSSION}

In this paper we have derived a nonlinear diffusion equation which describes the spatiotemporal evolution of cell number density in a one-dimensional cell-based model. It has been shown that the diffusion coefficient is proportional to the spring constant and inversely proportional to the viscosity coefficient. While these results are intuitively reasonable, the derivation allows cell-based parameters to be directly related to the macroscale population behavior. The diffusion coefficient has an inverse quadratic dependence on cell number density leading to deviation from standard Fickian behavior.

Comparisons of solutions of the cell-based and continuum models have shown excellent agreement. Initially, we derived a continuum equation in which cell position along an indexed chain was the dependent variable and used an analytic solution to confirm that the diffusion equation was a valid approximation to the cell-based simulations. In order to derive a more biologically intuitive continuum model, we made a coordinate transformation such that cell number density was the dependent variable, therefore putting the cellbased model in a framework which readily allows comparison with other models in the literature.

The derivation of continuum models from their discrete counterparts readily permits the classification of discrete model behaviors. This classification then allows one to choose which discrete model may be an appropriate model to choose for a given biological problem. In order to illustrate this point we compared the diffusion coefficient derived in this paper with that derived by Lushnikov et al. [23]. The comparison allows distinction to be drawn between discrete model behavior. Moreover, the underlying on- and off-lattice approaches considered by Lushnikov et al. [23] and in this paper, respectively, yield reaction diffusion equations for cell number density, raising the possibility that there are corresponding reaction diffusion equations for other comparable discrete models. The diffusion coefficients can be understood purely in terms of the respective underlying interaction rules and a distinction between on- and off-lattice does not appear to be important at the continuum level of description.

To demonstrate that the continuum framework derived here was robust to the inclusion of more realistic biological details, we considered a simple rule for cell division in the cell-based model. By choosing an appropriate source term in the continuum framework we were then able to demonstrate excellent agreement between the continuum and discrete models. In order that the nonlinear diffusion model can be used to model more realistic cell proliferation, it must be investigated how more detailed models of cell division at the cellular scale can be represented in a continuum framework.

As a result of transforming coordinates and describing the model in terms of cell number density, we have shown that in the limit of stiff springs, the model takes a similar form to that considered by Ward and King [16]. However, an interesting caveat of taking the limit is that the length scale over which diffusion acts must be much greater than that of the cell population, otherwise diffusion cannot act fast enough to make the population spatially homogeneous. While in the limit of stiff springs the model derived in this paper is qualitatively equivalent to that considered by Ward and King [16], the merit of deriving the model from first principles lies in the fact that we have defined limits in which the incompressibility assumption is acceptable. Moreover, the limits are defined in terms of parameters which are measurable at the cellular scale.

There are a number of questions yet to be answered about the form of the nonlinear diffusion coefficients. For example, one area of further investigation is whether or not the modeling methodology described in this paper can be applied to cell-based models in which the model parameters are nonhomogeneous. Moreover, dimensional arguments suggest that an analogous nonlinear diffusion model in 2D would be of the form $D(q)=\frac{\alpha}{q}$. However, in higher spatial dimensions there will not be such a natural cell ordering as in the onedimensional case, hence further approximations may be necessary to define a cell number density directly from the equations of motion. In order that the nonlinear diffusion model can be used as a realistic model of cell population evolution, it is necessary that corresponding models are derived in higher spatial dimensions. This topic will be explored in a future publication.

We finish by discussing how this "toy" model could be used to describe real tissues. In particular, one extension that we will explore in a future publication is that of intestinal crypts, which are test-tube-like indentations in the intestinal wall. Previously, these systems have been simulated $[9,17]$ using cell-based models in which cells interact via a linear force law in a cylindrical geometry. The continuum framework developed in this paper will allow us to much more efficiently understand model behavior in different regions of 
parameter space, as compared with a simulation-only approach. Moreover, a nondimensional analysis of the continuum model (similar to that presented in Sec. V B) will allow us to predict what regions of parameter space permit biologically realistic solutions.

The continuum model derived in this paper could be used to investigate the validity of considering a linear force law as a model for cell-cell interactions by, for example, measuring how an initially compressed epithelial cell population relaxes to an equilibrium configuration (see Fig. 7 for a schematic illustration). Choosing the spatial dimensions of the experiment such that boundary effects can be neglected over the time scale of interest, a symmetry argument suggests that the 1D model derived in this study could describe relaxation along the axis of compression. By tracking the boundary of the cell population using imaging analysis, the experimental results could be compared with simulation results such as those presented in Fig. 3, in order to (a) validate the choice of model and (b) experimentally determine the parameter $\alpha$ for a given cell line.

\section{ACKNOWLEDGMENTS}

P.J.M. and C.M.E. acknowledge the support of the Engineering and Physical Sciences Research Council through the Integrative Biology Project (Grant No. GR/S72023/01). P.J.M. also acknowledges training provided by the Oxford University Life Sciences Interface Doctoral Training Centre

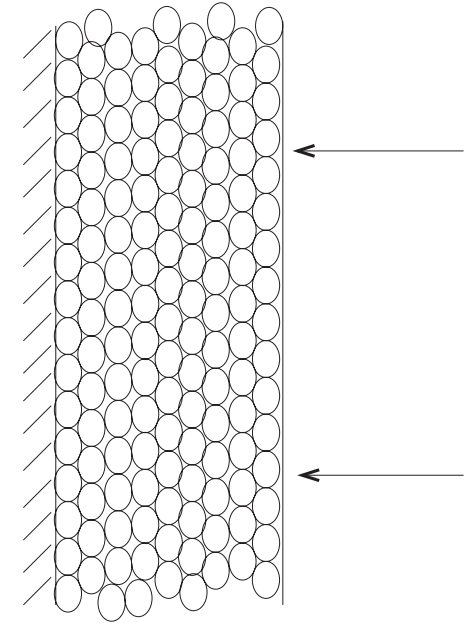

(a)

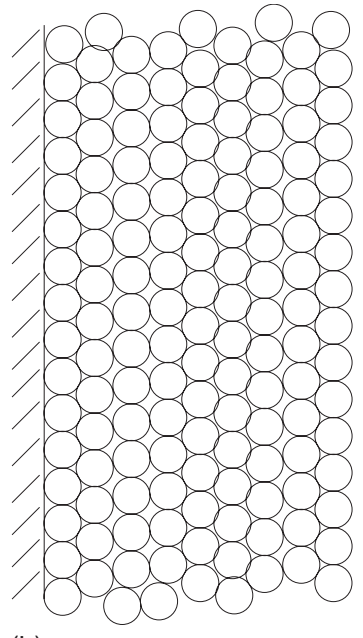

(b)
FIG. 7. (a) $t=0$; a schematic of a $2 \mathrm{D}$ monolayer of cells which is compressed against a fixed boundary. (b) $t=\infty$; upon removal of the compressing force the population of cells relaxes to an equilibrium configuration.

(LSI DTC). The authors would like to sincerely thank Radek Erban for helpful comments. Part of this work (P.J.M. and P.K.M.) was supported by NIH Grant No. U56CA113004 from the National Cancer Institute and the British Council through Grant No. PMI2. P.K.M. was partially supported by the Royal Society-Wolfson Foundation.
[1] W. Düchting and G. Dehl, J. Biomed. Eng. 2, 167 (1980).

[2] A. Deutsch and S. Dormann, Cellular Automaton Modeling of Biological Pattern Formation (Birkhauser, Boston, 2005).

[3] S. Dormann and A. Deutsch, In Silico Biol. 2 (3) (2002).

[4] F. Graner and J. A. Glazier, Phys. Rev. Lett. 69, 2013 (1992).

[5] M. Alber, N. Chen, P. M. Lushnikov, and S. A. Newman, Phys. Rev. Lett. 99, 168102 (2007).

[6] H. Honda, M. Tanemurab, and T. Nagaic, J. Theor. Biol. 228, 147 (2004).

[7] D. Drasdo and S. Hohme, Phys. Biol. 2, 133 (2005).

[8] J. Galle, M. Loeffler, and D. Drasdo, Biophys. J. 88, 62 (2005).

[9] F. Meineke, C. S. Potten, and M. Loeffler, Cell Prolif. 34, 253 (2001).

[10] G. Schaller and M. Meyer-Hermann, Phys. Rev. E 71, 051910 (2005).

[11] R. P. Araujo and D. L. S. McElwain, Bull. Math. Biol. 66, 1039 (2004).

[12] M. A. J. Chaplain, Math. Comput. Model. 23, 47 (1996).

[13] R. A. Gatenby and E. T. Gawlinski, Cancer Res. 56 (24) (1996).

[14] H. P. Greenspan, Stud. Appl. Math. 52 (1972).
[15] J. D. Murray, Mathematical Biology (Springer, New York, 1989).

[16] J. P. Ward and J. R. King, Math. Med. Biol. 14, 39 (1997).

[17] I. M. M. van Leeuwen et al., Cell Prolif. (unpublished).

[18] D. Drasdo, R. Kree, and J. S. McCaskill, Phys. Rev. E 52, 6635 (1995).

[19] J. Galle, G. Aust, G. Schaller, T. Beyer, and D. Drasdo, Am. J. Pathol. 169, 1802 (2006).

[20] J. R. King, J. Phys. A 23, 3681 (1990).

[21] A. A. Lacey, J. R. Ockendon, and A. B. Tayler, SIAM J. Appl. Math. 42, 1252 (1982).

[22] T. J. Newman, Math. Biosci. Eng. 2 (3)(2005).

[23] P. M. Lushnikov, N. Chen, and M. Alber, Phys. Rev. E 78, 061904 (2008).

[24] S. Turner, J. A. Sherratt, K. J. Painter, and N. J. Savill, Phys, Rev. E 69, 021910 (2004).

[25] J. C. Dallon and H. G. Othmer, J. Theor. Biol. 231, 203 (2004).

[26] P. E. Rouse, J. Chem. Phys. 21, 1272 (1953).

[27] P. G. de Gennes, Scaling Concepts in Polymer Physics (Cornell University Press, Ithaca, 1979).

[28] M. Doi and S. F. Edwards, The Theory of Polymer Dynamics (Oxford University Press, New York, 1986). 